BULLETIN OF THE

AMERICAN MATHEMATICAL SOCIETY

Volume 78, Number 4, July 1972

\title{
HARMONIC MAPPINGS OF SPHERES ${ }^{1}$
}

\author{
BY R. T. SMITH
}

Communicated by S. S. Chern, February 7, 1972

Introduction and statement of results. This announcement describes an elementary method of constructing harmonic maps in some cases not covered by the general existence theory. Recall that given smooth Riemannian manifolds $N$ and $M$, with $N$ compact, then the energy functional $E: H(N, M) \rightarrow R$ is defined on a suitable manifold of maps $H(N, M)$ and is given by $E(f)=\frac{1}{2} \int_{N}|d f|^{2}$. A map $f$ is said to be harmonic if $f$ is a critical point of $E$; equivalently, if the tension field $\tau(f)$ vanishes, where $\tau$ is the Euler-Lagrange operator associated to $E$.

The study of harmonic maps was initiated by Eells and Sampson, and the basic problem they consider is the following: given a homotopy class of maps between $N$ and $M$, is there a harmonic representative of that class? The case when $M$ is compact is of particular interest, and under the assumption that all sectional curvatures of $M$ are nonpositive, they succeeded in giving an affirmative answer to this question [2]. Their method was to find solutions of the heat equation $\tau(f)-\partial f / \partial t=0$ and obtain harmonic maps in the limit. More recently, the global theory of the calculus of variations has been successfully employed to recapture the existence theory [3], [4].

One may take the following as a starting point for the direct construction approach given here:

Observation. Let $f: R^{n+1} \rightarrow R^{m+1}$ be a map each of whose coordinates is given by a homogeneous harmonic polynomial of degree $k$. Suppose $f\left(S^{n}\right) \subseteq S^{m}$. Then $\bar{f}=f / S^{n}: S^{n} \rightarrow S^{m}$ is harmonic.

The examples of interest arise from putting two such maps together:

THEOREM. Let $f: R^{p} \rightarrow R^{q}$ and $g: R^{r} \rightarrow R^{s}$ be homogeneous harmonic polynomial maps of degree $l$ and $k$ respectively which send sphere to sphere. Assume

(1) $k>((\sqrt{2}-1) / 2)(r-2)$,

(2) $l>((\sqrt{2}-1) / 2)(p-2)$.

Further assume $r, p \geqq 2$. Then there is harmonic map $F: S^{p+r-1} \rightarrow S^{q+s-1}$ which is given in Euclidean coordinates by

(3) $F(u, v)=\left(\left(g(u) /|u|^{k}\right) \cos \alpha(t),\left(f(v) /|v|^{l}\right) \sin \alpha(t)\right)$

where $t=\log (|u| /|v|)$ and $\alpha$ is a smooth monotone function on the reals which

AMS 1970 subject classifications. Primary 53C99; Secondary 58G99.

Key words and phrases. Harmonic mapping, harmonic polynomial.

1 This research comprises part of the author's dissertation at Warwick University and was supported by a Marshall Scholarship. 
is asymptotic to 0 at $t=-\infty$ and asymptotic to $\pi / 2$ at $t=\infty$. A modified formulation holds if $r=1$ or $p=1$.

CoROLlary. Let $g$ be as above, with (1) satisfied. Then the ith suspension of the map of spheres defined by $g$ can be harmonically realized for $i=$ $1, \ldots, 6$.

EXAMPLES. (a) Let $g: \boldsymbol{C} \rightarrow \boldsymbol{C}$ be $z \longmapsto z^{k}$. Suspension gives harmonic maps of $S^{n}$ to $S^{n}$ of degree $k$ for $n=2, \ldots, 7$.

(b) Let $g$ be the harmonic polynomial map which defines the Hopf map of $S^{3}$ to $S^{2}$. Suspension gives essential harmonic maps of $S^{n+1}$ to $S^{n}$ for $n=3, \ldots, 8$.

(c) Similarly for the $g$ which gives the Hopf map of $S^{7}$ to $S^{4}$. Suspensions yield essential harmonic maps of $S^{n+3}$ to $S^{n}$ for $n=5, \ldots, 10$. Furthermore, if we take $f: C \longrightarrow C$ to be $z \longmapsto z^{k}$, then $f$ and $g$ can be combined to get harmonic representatives for $\pi_{9}\left(S^{6}\right)=Z_{24}$.

It is not yet possible to suspend (harmonically) the last Hopf map, due to requirement (1); that this map is itself harmonic follows from the Observation, or otherwise. For the sake of completeness, also note the following:

Observation. Let $g: S^{2} \longmapsto S^{2}$ be $z \longmapsto z^{k}$ on the Riemann sphere and let $h: S^{3} \rightarrow S^{2}$ be the standard form of the Hopf map. Then $g \circ h$ is harmonic (and has Hopf invariant $k^{2}$ ).

Remarks. (1) The Morse index of these maps (considered as critical points of the energy) has not yet been computed. However, it is certainly expected that the index of those of degree $k$ on $S^{n}$ will be positive $(n \geqq 3)$ since the energy takes arbitrarily small values in each component of the mapping space [2]. Incidentally, what can be shown is that index $\left(\mathrm{id}_{s^{2}}\right)=0$ and index $\left(\mathrm{id}_{S^{n}}\right)=n+1$ for $n \geqq 3$, using known facts about the spectrum of $S^{n}$.

(2) Although this announcement only discusses essential harmonic maps, there is ample opportunity here for constructing others which are inessential but geometrically interesting. For example, the standard nontrivial minimal immersions of $S^{n}$ in higher spheres are given by harmonic polynomials.

I would like to take this opportunity to thank my official and unofficial supervisors David Elworthy and James Eells for their valuable help throughout this project, and also to express gratitude to Larry Markus and David Chillingworth for their insight on differential equations.

Methods. The problem is reduced to analyzing an ordinary differential equation for the function $\alpha$ of the Theorem. That such a reduction is possible is rather special to the polynomial framework; even suspensions 
of harmonic maps are not easily handled in general. The study of this equation is then facilitated by a physical analogy.

Firstly, note that if $f: M \rightarrow S^{n}$ is a map of a Riemannian manifold $M$, then $f$ is harmonic if and only if the induced map $f: M \rightarrow R^{n+1}$ satisfies

$$
\Delta f-|d f|^{2} f=0 .
$$

Here $\Delta$ is the Laplacian of $M$ for functions. Applying this to (3) yields the necessary condition

$$
\begin{aligned}
\ddot{\alpha}(t)+\frac{1}{e^{t}+e^{-t}} & \left(\left((r-2) e^{-t}-(p-2) e^{t}\right) \dot{\alpha}(t)\right. \\
& \left.+\left(l(l+p-2) e^{t}-k(k+r-2) e^{-t}\right) \frac{\sin 2 \alpha(t)}{2}\right)=0 .
\end{aligned}
$$

Thinking of $t$ as a time parameter, we see that (5) is the equation of motion of some sort of pendulum. Notice that "gravity" is positive for $t \gg 0$ and negative for $t \ll 0$, so that one can look for an exceptional trajectory in which the pendulum is standing on end at $t=\infty\left(\alpha_{\infty}=\pi / 2\right)$ and hanging straight down at $t=-\infty\left(\alpha_{-\infty}=0\right)$.

The existence of such a solution is established in the intuitively obvious way. Fix $t_{0}$ to be the time when gravity vanishes and manipulate the initial conditions $\alpha_{0}$ and $\dot{\alpha}_{0}$. For a given $\alpha_{0} \in(0, \pi / 2)$, throw the pendulum just hard enough $\left(\dot{\alpha}_{0}=\dot{\alpha}_{0}^{+}\left(\alpha_{0}\right)\right)$ that $\alpha(t) \sim \pi / 2$ as $t \rightarrow \infty$; similarly, choose $\dot{\alpha}_{0}^{-}\left(\alpha_{0}\right)$ to get $\alpha(t) \sim 0$ as $t \rightarrow-\infty$. Then $\dot{\alpha}_{0}^{+}$and $\dot{\alpha}_{0}^{-}$are continuous in $\alpha_{0}$. Further, $\dot{\alpha}_{0}^{+} \rightarrow 0$ as $\alpha_{0} \rightarrow \pi / 2$ and $\dot{\alpha}_{0}^{-} \rightarrow 0$ as $\alpha_{0} \rightarrow 0$. On the other hand, to show that $\dot{\alpha}_{0}^{+}$is bounded away from 0 for $\alpha_{0}$ near 0 (and that $\dot{\alpha}_{0}^{-}$is bounded away from 0 for $\alpha_{0}$ near $\pi / 2$ ) requires recourse to a second order comparison theorem [1, p. 210] and essential use of the fact that (5) is underdamped for large $|t|$, using (1) and (2). Having done this, it follows that there is some $\alpha_{0}$ such that $\dot{\alpha}_{0}^{+}\left(\alpha_{0}\right)=\dot{\alpha}_{0}^{-}\left(\alpha_{0}\right)$, and existence is proved.

REMARK. The unpleasantly restrictive assumptions (1) and (2) occur crucially only here, and it is not known to what extent they might be circumvented. For example, if (5) is symmetrical, with $k=1$ and $p=r$, they can be ignored.

There remains the difficult problem of regularity. It must be shown that the function $F$ actually extends to a $C^{2}$ mapping of the sphere. This requires estimating the asymptotic behavior of $\alpha$. Having done this (via a series of ad hoc comparison theorems), one can demonstrate the required smoothness of $F$ on $R^{p+r}-(0)$ by exercising suitable care.

REMARKS. With reference to the Observation regarding $\pi_{3}\left(S^{2}\right)$, it is to be noted that the most natural direct approach to the remaining homotopy classes fails. The relevant equation is quite stable, but a nonconservation 
of energy argument shows that no solution of the desired form exists. For this reason, the existence or nonexistence of a harmonic map of Hopf invariant 2 is an interesting unresolved case.

The pendulum approach has further applicability, for example to two-dimensional toroids and ellipsoids of revolution. Harmonic maps of degree $k$ in the latter case will be holomorphic with respect to the induced complex structure.

\section{REFERENCES}

1. E. A. Coddington and N. Levinson, Theory of ordinary differential equations, McGrawHill, New York, 1955. MR 16, 1022.

2. J. Eells and J. H. Sampson, Harmonic mappings of Riemannian manifolds, Amer. J. Math. 86 (1964), 109-160. MR 29 \# 1603.

3. H. I. Eliasson, Variation integrals in fiber bundles, Proc. Sympos. Pure Math., vol. 16, Amer. Math. Soc., Providence, R.I., 1970, pp. 67-89. MR 42 \# 2507.

4. K. Uhlenbeck, Harmonic maps: A direct method in the calculus of variations, Bull. Amer. Math. Soc. 76 (1970), 1082-1087. MR 41 \#9305.

Department of Mathematics, University of Warwick, Coventry, Warwickshire CV4 7AL, ENGLAND 